\title{
Biofilm production by uropathogens causing catheter associated urinary tract infection
}

\author{
Tomar R. ${ }^{1}$, Paviaya R. ${ }^{2}$, Ghosh S. ${ }^{3}$, Murthy R. ${ }^{4}$, Pradhan S. ${ }^{5}$, Agrawal E. ${ }^{6}$ \\ ${ }^{1}$ Dr Rashmi Tomar, Assistant Professor, Department of Microbiology, Chhattisgarh Institute of Medical \\ Sciences Bilaspur, CG, India. ${ }^{2}$ Dr Rajendra Singh Paviaya, Assistant Professor, Department of Physiology, \\ Chhattisgarh Institute of Medical Sciences Bilaspur, CG, India. ${ }^{3}$ Dr Mrs S. J Ghosh, Associate Professor and \\ Head, Department of Microbiology, Dr. D. Y. Patil Medical College, Kolhapur, India. ${ }^{4}$ Dr Ramanesh Murthy, \\ Professor and Head of Department of Microbiology, Chhattisgarh Institute of Medical Sciences Bilaspur, CG, \\ India. ${ }^{5}$ Dr Sagarika Pradhan, Assistant Professor, Department of Microbiology, Chhattisgarh Institute of \\ Medical Sciences Bilaspur, CG, India. ${ }^{6}$ Dr Ekta Agrawal, Assistant Professor, Department of Microbiology, \\ Chhattisgarh Institute of Medical Sciences Bilaspur, CG, India.
}

Address for Correspondence: Dr. Rajendra Singh Paviaya, Assistant Professor, Department of Physiology, Chhattisgarh Institute of Medical Sciences Bilaspur, CG, India. Email:drrspawaiyaa@yahoo.com

\begin{abstract}
Background: The microbial populations within urinary catheter frequently develop as biofilms, directly attaching to the surface of catheters. Bacteria in biofilm are protected from antimicrobial chemotherapy as well as host defence mechanisms, establishing chronic persistent infections, septicemia and death if not treated. Material and Method: The present study, includes 200 patients, catheterized for $>48$ hours at CIMS, Bilaspur. Urine samples were collected and inoculated in nutrient agar, blood agar and MacConkey agar plates and identification done as per standard procedure. This study was conducted to detect biofilm formation ability of uropathogens by two different methods (Tube and Congo red agar method) and compare their antibiotic sensitivity by using Kirby-Bauer disc diffusion method. Results: Out of 200 urine samples significant bacteriuria were detected in $148(74 \%)$ of samples and no growth found in $52(26 \%)$ samples and 14 samples showed growth of 2 microorganisms. A total no. of 162 microorganisms were isolated from 200 urine samples. Among these 162 isolates E. Coli was 29.62\%, Pseudomonas aeruginosa $11.72 \%$, Klebsiella sp $18.51 \%$, Citrobactor sp 7.40\%, Staphylococcus aureus 3.08\%, and Coagulase negative Staphylococci 11\%. Among these 162 isolates, a total of $91(56.17 \%)$ isolates showed biofilm production. Percentage of biofilm formation was highest in P. aeruginosa (63.15\%). Conclusion: Tube test method was found to be more reliable method. The in vitro antibiotic susceptibility pattern of biofilm producing organisms showed less sensitivity as compared to non-biofilm producing organisms.
\end{abstract}

Keywords: Biofilm, Catheter associated urinary tract infection, Antibiotic resistance

\section{Introduction}

"A biofilm is an aggregate of micro-organisms in which cells adhere to each other on a surface embedded within a self-produced matrix of extracellular polymeric substance"[1]. Urinary tract infection, with its diverse clinical syndromes and affected host groups, remains one of the most common but widely misunderstood and challenging infectious diseases encountered in clinical practice.

Manuscript received: $26^{\text {th }}$ June 2017

Reviewed: $5^{\text {th }}$ July 2017

Author Corrected: $14^{\text {th }}$ July 2017

Accepted for Publication: $21^{\text {st }}$ July 2017
The risk of developing urinary tract infection increases significantly with the use of indwelling devices such as catheters and urethral stents or sphincters. Urinary tract infections account for an estimated 25 to $40 \%$ of nosocomial infections and represent the most common type of these infections [2]. Catheter associated urinary tract infections (CAUTI) account for up to $40 \%$ of all nosocomial infections and $80 \%$ of all nosocomial Urinary Tract Infections (UTIs) [3]. 
The organisms commonly contaminating these devices are Escherichia coli, Proteus mirabilis, Pseudomonas aeruginosa, Klebsiella pneumoniae, Staphylococcus epidermidis and Enterococcus faecalis. The longer the urinary catheter remains in place, the greater the tendency of these organisms to develop urinary tract infections [2].

Organisms causing CAUTIs require fewer recognized virulence factors to colonize and establish infection, including bacterial adhesions, degradative enzymes, toxins and capsules, lipopolysaccharides. These virulence factors help bacteria to attach on catheter surface and to induce an inflammatory response. Biofilm is one of the most important virulence factor of bacteria which causes CAUTIs [4].

Most cases of CAUTI are associated with biofilm formation which is a representative type of biofilm associated infection usually composed of multi drug resistant microorganisms [2].

\section{Materials and Methods}

Study design- Cross Sectional study

Study setting- Department of Microbiology, Chhattisgarh institute of Medical Science, Bilaspur, over a period of one year

Inclusion criteria- All ages groups, both Sexes, who indwelling urinary catheter for at least 2 days, and who were suffering from symptoms of UTIs

Exclusion criteria- patients with symptoms of UTI prior to catheterization

Participants- After approval from ethical committee, samples collected from admitted patients from Chhattisgarh institute of medical science hospital

Study size- 200 Samples were inoculated in nutrient agar, blood agar and MacConkey agar plates, as per standard procedure [5].

After 24 hours of incubation at $37^{\circ} \mathrm{C}$ in aerobic atmosphere, inoculated plates were observed for presence of growth and a colony count was done. The results were recorded as positive where urine culture showed colony count of 100 or more which was equivalent to $10^{5} \mathrm{CFU} / \mathrm{ml}[5]$.

After identification on the basis of colony morphology, gram stain and motility test, the isolates were subjected to biochemical tests for species identification [6].

Biofilm formation was detected by Congo red agar method and Tube test method.

Congo Red Agar Method- CRA medium proposed by Freeman et al. was prepared with brain heart infusion broth, sucrose, agar and Congo red indicator. Congo red stain was prepared separately in sterile distilled water and was added to sterile molten agar base and then the medium was poured in the plates. CRA plates were inoculated with the test organisms and incubated at $37^{\circ} \mathrm{C}$ for 24 hours to 48 hours aerobically. Black colonies with dry crystalline consistency indicate strong biofilm formation. Brownish or reddish growth was considered as negative biofilm formation (Fig. 1) [7].

Tube Method- Biofilm production was investigated by the tube adherence test proposed by Christensen et al. Ten $\mathrm{ml}$ Trypticase soya broth with $1 \%$ glucose was inoculated with the test organism on nutrient agar individually. Broths were incubated at $37{ }^{\circ} \mathrm{C}$ for 24 hours. The cultures were aspirated and the tubes were washed with phosphate buffer saline $\mathrm{pH}$ 7.3. The tubes were dried and stained with $0.1 \%$ crystal violet. Excess stain was removed. Tubes were dried in inverted position. In positive biofilm formation, a visible stained film was seen along the walls and bottom of the tube (Fig. 2) [7].

All isolates were subjected to antibiotics susceptibility testing on Mueller Hinton agar by disc diffusion method of Kirby-Bauer using commercially available discs from Himedia [8]. 


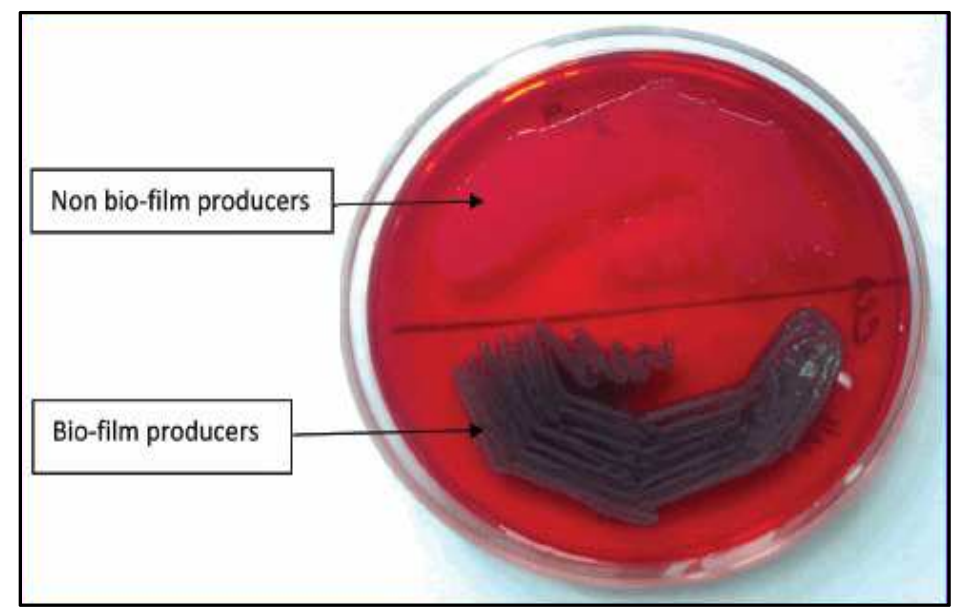

Fig-1: Congo red method for biofilm detection

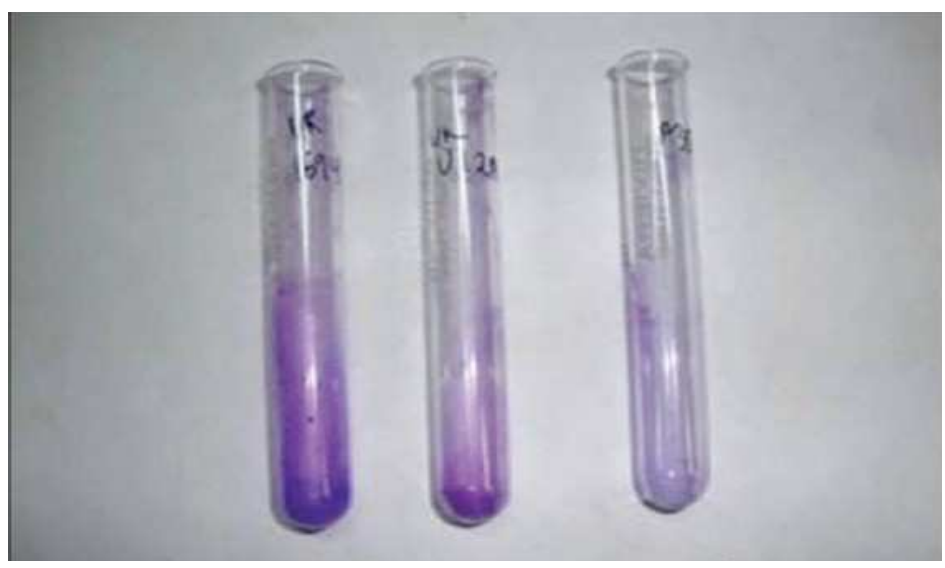

Fig-2: Tube test method for biofilm detection

\section{Results}

Out of 200 urine samples significant bacteriuria was detected in 148 (74\%) of samples and no growth was found in $52(26 \%)$ samples.

As shown in the table 1, rate of developing UTI was more with increase in duration of catheterization and it was $87.5 \%$ with (7-14 days) of catheterization.

Table-1: Length of catheterization in relation to development of significant bacteriuria.

\begin{tabular}{|c|c|c|}
\hline Duration & No. Of Samples & Significant bacteriuria \\
\hline$(2-7$ days $)$ & 20 & $3(15 \%)$ \\
\hline$(7-14$ days $)$ & 72 & $63(87.5 \%)$ \\
\hline$(>14$ days $)$ & 8 & $6(75 \%)$ \\
\hline Total & $\mathbf{1 0 0}$ & $\mathbf{7 2}$ \\
\hline
\end{tabular}

Among these 148 positive samples, 14 samples showed growth of 2 organisms. A total no. of 162 microorganisms were isolated from 200 urine samples.

As shown in the table 2, E. coli was found to be most frequently isolated pathogen $(29.62 \%)$ followed by Klebsiella sp. (18.51\%) and enterobactor (13.58\%). 
Table-2: Distribution of uropathogens from urine samples

\begin{tabular}{|c|c|c|}
\hline Name of Bacteria & No. & Percentage (\%) \\
\hline Escherichia coli & 48 & 29.62 \\
\hline Klebsiella species & 30 & 18.51 \\
\hline Enterobactor & 22 & 13.58 \\
\hline Pseudomonas aeruginosa & 19 & 11.72 \\
\hline Enterococci & 15 & 9.25 \\
\hline Citrobacter freundii & 12 & 7.40 \\
\hline Coagulase -ve Staphylococci & 11 & 6.79 \\
\hline Staphylococcus aureus & 5 & 3.08 \\
\hline Total & $\mathbf{1 6 2}$ & $\mathbf{1 0 0}$ \\
\hline
\end{tabular}

Table-3: Biofilm formation among these urinary isolates

\begin{tabular}{|c|c|c|}
\hline Organisms & Biofilm positive & $\begin{array}{c}\text { Biofilm } \\
\text { negative }\end{array}$ \\
\hline P. aeruginosa (19) & 12 & 7 \\
\hline Klebsiella sp (30) & $18(60 \%)$ & $12(40 \%)$ \\
\hline Enterococci(15) & 9 & 6 \\
\hline E. $\operatorname{coli}(48)$ & $28(58.33 \%)$ & $20(41.66 \%)$ \\
\hline Enterobactor(22) & 12 & 10 \\
\hline Citrobactor sp(12) & 6 & 6 \\
\hline S. aureus(5) & 2 & 3 \\
\hline CONS(11) & 4 & 7 \\
\hline Total(162) & $91(56.17 \%)$ & $71(43.82 \%)$ \\
\hline
\end{tabular}

A total of $91(56.17 \%)$ isolates showed biofilm formation by Congo red or Tube test. Percentage of biofilm formation was highest in P. aeruginosa (63.15\%) followed by Klebsiella sp (60\%) and E. coli (58.33\%). [Table $3]$

Tube test methods detected $77.8 \%$ biofilm positive isolates whereas Congo red test detected $71.1 \%$ isolates producing biofilm.

The in vitro antibiotic susceptibility pattern of gram negative organisms showed very less sensitivity to gentamycin $(29.1 \%)$, tobramycin $(45.8 \%)$, amoxyclav $(37.5 \%)$, ciprofloxacin $(20.8 \%)$, doxycycline $(33.3 \%)$, ceftriaxone $(37.5 \%)$, ceftazidime $(41.7 \%)$ and levofloxacin $(37.5 \%)$.

Sensitivity to amikacin (66.6\%), nitrofurantoin (70.8\%) piperacillin - tazobactum (83\%), imipenam and meropenam $(87.5 \%)$ were good.

The in vitro antibiotic susceptibility pattern of biofilm producing Gram negative organisms showed less sensitivity as compared to non-biofilm producing organism such as gentamycin $(23.68 \%)$, tobramycin $(31.5 \%)$, amoxyclav (31.5\%), ciprofloxacin (10.5\%), doxycycline (23.68\%), ceftriaxone (28.9\%), and ceftazidime (36.8\%). Among biofilm producing isolates, four isolates of Pseudomonas, two isolates of Klebsiella and two isolates of E.coli showed resistant to all drugs whereas in biofilm non-producing isolates resistant to all drugs were found only in one each isolates of Klebsiella species and E. coli.

Staphylococcal species were less sensitive to penicillin (0\%), ampicillin (0\%), levofloxacin (27.7\%), ciprofloxacin (27.7\%), ofloxacin (33.33\%), norfloxacin (33.33\%), doxycycline (38.9\%), and cotrimoxazole (38.9\%). Staphylococcal species showed good sensitivity to linezolid (88.8\%) and nitrofurantoin $(66.6 \%)$. 


\section{Discussion}

Biofilm, a predominant mode of growth characteristics of bacteria, plays a central role in pathogenesis of catheter associated urinary tract infection [9].

Urinary catheterization is generally indicated to relieve urinary tract obstruction, to permit urinary drainage in patients with neurogenic bladder dysfunction and urinary retention, to aid urologic surgery and to obtain accurate measurement of urinary output in clinically ill patients. An estimated 4 million patients are subjected yearly to urinary catheterization and therefore at risk for catheter associated infection and its related sequelae [10]. In the present study, $74 \%$ of the 200 patients studied had significant bacteriuria, and $26 \%$ samples were culture negative.

As shown in table 4, our result is similar with study conducted by Asha B Patil et al [12].

Table-4: Incidence of CAUTI in various studies.

\begin{tabular}{|c|c|c|}
\hline Study series & Year & Incidence of CAUTI \\
\hline Rohan Chaudhari et al [20] & 2004 & $44 \%$ \\
\hline Taiwo SS et al10 & 2006 & $88.5 \%$ \\
\hline Onipede Anthony et al [16] & 2010 & $60.9 \%$ \\
\hline S. Abaeze et al [14] & 2011 & $41.10 \%$ \\
\hline Mahabulbul Ishlam et al [11] & 2014 & $90 \%$ \\
\hline Asha B Patil et al [12] & 2014 & $76 \%$ \\
\hline Present study & 2014 & $74 \%$ \\
\hline
\end{tabular}

In present study rate of developing UTI was more with increase in duration of catheterization which is similar to reports of S G Kulkarni et al [13], Taiwo SS et al [10] and Mahabulbul Ishlam et al [11].

The distribution of commonest bacterial organisms in the present study is close to results obtained by G F M Gad et al [17] and S. Abaeze et al [14]. E. coli was most frequently isolated organism $48(29.62 \%)$ and this is similar to study conducted by S. Abaeze et al [14], Mahabulbul Ishlam et al[11], Asha B Patil et al [12] and S. Niveditha 2012 et al [15].

Implanted prosthetic devices constitute particularly attractive surfaces for bacterial colonization, as they have none of the protective mechanisms of healthy tissue surfaces.

As shown in table 5, Our results are similar with S. Niveditha et al [15].

Table-5: Detection of biofilm formation in various studies.

\begin{tabular}{|c|c|}
\hline Various studies & Biofilm formation \% \\
\hline G Reid et al [21] & $73 \%$ \\
\hline Narmeen Mahmoud et al [22] & $43.3 \%$ \\
\hline S. Niveditha et al $[15]$ & $60 \%$ \\
\hline Pradeep kumar et al $[9]$ & $80 \%$ \\
\hline Present study & $56.17 \%$ \\
\hline
\end{tabular}

The percentage of biofilm detection of different isolates in the present study is similar to reports of S. Niveditha et al [15]. In the present study Tube test method was more reliable. Afreenish Hassan et al [7] also found that tube method is more reliable than congo red agar method.

In the present study sensitivity pattern of non-biofilm producing $E$. coli is similar to study conducted by Mahabulbul Ishlam et al [11]. 
Sensitivity pattern of Klebsiella species in present study is smilar to reports of Onipede Anthony et al [16], Taiwo SS et al [10] and S G Kulkarni et al [13]. Sensitivity pattern of P. aeruginosa in the present study is similar to reports of S G Kulkarni et al [13] and S.Abaeze et al [14]. In the present study sensitivity of $C$. freundii is similar to results obtained by Onipede Anthony et al [16].

Our study showed that imipenam, meropenam, nitrofurantoin, amikacin and piperacillin-tazobactam were most effective antibiotics against gram negative isolates. Narmeen Mahmoud et al [19] also found that imipenam and amikacin were most effective antibiotics against gram negative isolates.

In the present study cefotaxime, ceftriaxone, norfloxacin, ciprofloxacin, ofloxacin and the aminoglycosides showed less sensitivity against gram negative isolates. The present study showed more drug resistance in biofilm forming isolates than in non bioflim forming isolates which is similar to S. Pramodhini et al [18].

Our study showed that, resistance to all drugs were found more in biofilm positive isolates then in biofilm non producing isolates which is similar to reports of Mahabulbul Ishlam et al [11]. In the present study sensitivity pattern of Staphylococci is similar to reports of others.

\section{Conclusion}

Indwelling urethral catheters should be avoided whenever possible and should never be resorted to unless with absolute indications.

There is an association between biofilm production with persistent CAUTI and antibiotic therapy failure. Hence identification of infection caused by biofilm producing organisms might help to modify the antibiotic therapy and prevent infection.

In our view this is the first of its kind highlighting role and relevance of biofilm production by pathogenic bacteria isolated from catheter associated urinary tract infection in this tribal dominated region of Chhattisgarh state.

\section{Funding: Nil, Conflict of interest: None Permission of IRB: Yes}

\section{References}

1. What are Biofilms? Montana State University, Center for Biofilm Engineering: Biofilm research \& education relevant to industry, health, and the environment. Website available at: www.biofilm. montana. edu/ biofilm-basics.html

2. Drekonja DM, Johnson JR. Urinary tract infections. Prim Care. 2008 Jun;35(2):345-67, vii. doi: 10.1016/j.pop.2008.01.001.

3. Trautner BW. Management of catheterassociated urinary tract infection. Curr Opin Infect Dis. 2010 Feb; 23(1):76-82. doi: 10.1097/QCO. 0b013e328334dda8.
4. Oelschlaeger TA, Dobrindt U, Hacker J. Virulence factors of uropathogens. Curr Opin Urol. 2002 Jan; 12(1):33-8.

5. Forbes BA,Sahm DF, Weissfield AS. Bailey and Scott's Diagnostics Microbiology, $10^{\text {th }}$ ed, St. Louis, Misssouri, USA: Mosby Inc.;1998.

6. MacFaddin J. Biochemical Tests for Identification of Bacteria. $3^{\text {rd }}$ ed. Philadelphia: Lippincott Williams and Wilkins; 1976.

7. Afreenish Hassan, Javaid Usman, Fatima Kaleem, Maria Omair, Ali Khalid, Muhammad Iqbal. Evaluation of different detection methods of biofilm formation in the clinical isolates, Braz $\mathrm{J}$ Infect Dis 2011;15 (4)

8. Clinical and Laboratory Standard Institute. Performance Standards for Antimicrobial Suseptibility Testing. Vol. 1, No. 1, M2 A9. Pennsylvania, USA: Clinical and Laboratory Standard Intitute; 2007.

9. Pradeep Kumar SS, Easwer HV, Maya Nandkumar A. Multiple drug resistant bacterial biofilms on implanted catheters - a reservoir of infection. J Assoc Physicians India. 2013 Oct; 61 (10) : 702-7.

10. Taiwo SS and Aderounmu AOA. Catheter Associated Urinary Tract Infection: Aetiologic Agents and Antimicrobial Susceptibility Pattern in Ladoke Akintola University Teaching Hospital, 
Osogbo, Nigeria. African Journal of Biomedical Research, 2006; 9: 141 - 148

11. Mahabubul Islam Majumder, Tarek Ahmed, Delwar Hossain, Mohammad Ali, Belalul Islam and Nazmul Hasan Chowdhury. Bacteriology and Antibiotic Sensitivity Patterns of Urine and Biofilm in Patients with Indwelling Urinary Catheter in a Tertiary Hospital in Bangladesh, J Bacteriol Parasitol 2014, 5:3

12. Asha B Patil, Shobha D Nadagir, Rashmi Dhaduti, Praveen AJ, Syeda Fasiha Mohammadi, Lakshminarayana SA, Namratha WN.Catheterassociated urinary tract infection: Aetiology, ESBL production, and risk factors. JIACM 2014; 15(1): 22-51.

13. Dr. S. G.Kulkarni1, Dr. S. H. Tali, Dr. Manjiri Naik, Dr. Amit Kale. Profile of Urinary Tract Infection in Indwelling Catheterized Patients. Journal of Dental and Medical Sciences 2014;13 (4): 132-138.

14. S. Abaeze et al., The prevalence of urinary catheter related infections in federal medical centre Abeokuta Nigeria. Int J Pharm Biomed Sci 2011, 2 (3), 81-85.

15. S. Niveditha, S. Pramodhini, S. Umadevi, Shailesh Kumar, and Selvaraj Stephen .The Isolation and the Biofilm Formation of Uropathogens in the Patients with Catheter Associated Urinary Tract Infections (UTIs). J Clin Diagn Res. 2012 November; 6(9): 1478-1482.

16. Onipede Anthony, Oyekale T Oluwalana, Olopade Bolatito, Olaniran Olaniran, Oyelese Adesola, and Ogunniyi Titus. Urinary Pathogens and Their Antimicrobial Susceptibility in Patients with Indwelling Urinary Catheter. Sierra Leone Journal of Biomedical Research. June, 2010 Vol. 2 (1) pp. 47-53.
17. Gamal Fad Mahmoud Gad, Mohamed Ali ElFeky, Mostafa Said El-Rehewy, Mona Amin Hassan, Hassan Abolella, and Rehab Mahmoud Abd El-Baky.Detection of icaA, icaD genes and biofilm production by Staphylococcus aureus and Staphylococcus epidermidis isolated from urinary tract catheterized patients.J Infect Dev Ctries 2009; 3(5): 342-351.

18. S. Pramodhini,S. Niveditha, S. Umadevi, Shailesh Kumar, and Selvaraj Stephen,Antibiotic resistance pattern of biofilm forming uropathogens isolated from catheterized patients in Pondichery, India. Australasian Medical Journal AMJ 2012,5 (7) $344-348$.

19. Nermeen Mahmoud Ahmed Abdallah, Shereen Bendary Elsayed, Manal Mohamed Yassin, Mostafa and Ghada Metwally El-gohary. Biofilm forming bacteria isolated from urinary tract infection, relation to catheterization and susceptibility to antibiotics. International Journal for Biotechnology and Molecular Biology Research 2011; 2(10): 172-178.

20. Rohan Chaudhari, Anjali Deshpande, Subhash A Angadi and Geeta V Koppikar (2004): A Preliminary Study of Catheter Associated Bacteriuria, Bombay hospital journal. 2004; 46 (03)

21. G Reid PhD, R Charbonneau-Smith RN, D Lam MSC, Yun Suk Kang, student, M Lacerte MD, $\mathrm{KC}$ Hayes $\mathrm{PhD}$. Bacterial biofilm formation in the urinary bladder of spinal cord injured Patients. Paraplegia 30 1992; 711-71.

22. Henry F. Alavaren, Jodor A. Lim, Melecia Antonio-Velmonte, and Myrna T. Mendoza, Urinary Tract Infection in Patients with Indwelling Catheter. Phil J Microbiol Infect Dis 1993; 22(2): 65-74.

\section{How to cite this article?}

Tomar R, Paviaya R, Ghosh S, Murthy R, Pradhan S, Agrawal E. Biofilm production by uropathogens causing catheter associated urinary tract infection. Int J Med Res Rev 2017;5(07):710-716.doi:10.17511/ijmrr. 2017.i07.09. 